\title{
Tracer-Encapsulated Solid Pellet (TESPEL) Injection System for Wendelstein 7-X)
}

\author{
R. Bussiahn, ${ }^{1, b)}$ N. Tamura, ${ }^{2}$ K.J. McCarthy, ${ }^{3}$ R. Burhenn, ${ }^{1}$ H. Hayashi, ${ }^{2}$ R. Laube, ${ }^{1}$ T. Klinger, ${ }^{1}$ the LHD \\ experiment group, ${ }^{2, c)}$ and the W7-X team ${ }^{1, d}$ ) \\ 1) Max-Planck-Institut für Plasmaphysik, Greifswald, Germany \\ ${ }^{2)}$ National Institute for Fusion Science, National Institutes of Natural Sciences, Toki, \\ Japan \\ ${ }^{3)}$ Centro de Investigaciones Energéticas, Medioambientales y Tecnológicas, Madrid, \\ Spain
}

Impurity confinement in fusion plasmas is mainly determined by transport mechanisms in the core region. For the Wendelstein 7-X stellarator, its island divertor is expected to screen effectively external impurity sources in the scrape-off layer at higher densities. However, the unique feature of Tracer-Encapsulated Solid Pellet (TESPEL) injection, releasing impurities at a well-localized radial position directly in the core plasma enables investigating such transport mechanisms. This paper reports on the detailed design of a completely new TESPEL injection system, which has been designed by the National Institute for Fusion Science, Toki, Japan and is currently being installed at Max-Planck-Institut für Plasmaphysik, Greifswald, Germany, for the Wendelstein 7-X. This injector consists of a storage and injection unit, attached to a system of guiding tubes which run through 3 successive differential pumping stages. A light-gate system and an optical observation system are used to determine the location of the deposited tracers. Laboratory tests, carried out by shooting TESPELs onto a sample foil showed its good performance after careful realignment of the guiding tubes.

\section{INTRODUCTION}

Impurity transport is one of several critical issues that need to be fully elucidated on the pathway to developing a high-temperature magnetically confined fusion reactor. In order to facilitate impurity transport studies in magnetically confined plasmas, the Tracer-Encapsulated Solid Pellet (TESPEL) ${ }^{1}$ method was developed by the National Institute for Fusion Science in Japan. TESPEL is a double-layered impurity pellet. It consists of a polystyrene $\left(\mathrm{C}_{8} \mathrm{H}_{8}\right)_{n}$ polymer outer layer with a selected impurity tracer embedded in its core. The tracer amount in the TESPEL is deduced during the fabrication process by measuring the particle size. Due to its structure, a TESPEL protects its tracer impurity during early phases of plasma interaction and deposits it then within a three-dimensionally limited region within the plasma. ${ }^{2}$. To date, TESPEL experiments have been undertaken in the CHS, the LHD and TJ-II stellarator devices ${ }^{3-5}$. For instance, LHD experiments highlighted the importance of the location of the impurity source (inside or outside the last-closed flux surface) on impurity behavior within the plasma ${ }^{4}$. Also, the use of a TESPEL system on TJII has resulted in broadening of current knowledge on pellet ablation and particle deposition ${ }^{6}$. Moreover, such results reinforce the importance of undertaking comprehensive impurity transport studies on different devices

\footnotetext{
a) Published as part of the Proceedings of the 22nd Topical Conference on High-Temperature Plasma Diagnostics (HTPD 2018) in San Diego, California, USA.

b) Electronic mail: rene.bussiahn@ipp.mpg.de

c) Y. Takeiri et al., Nucl. Fusion 57, 102023 (2017)

d) R. C. Wolf et al., Nucl. Fusion 57, 102020 (2017)
}

with a common experimental tool. This would be of significant benefit as it would help reduce ambiguity in the results.

In this paper the authors report on a new TESPEL system that has been developed for the recently commissioned Wendelstein 7-X (W7-X) stellarator. In Sec. II, the W7-X stellarator, its TESPEL injection and its associated diagnostics, are described. In Sec. III, first results from laboratory testing of this TESPEL injection system on a test bed are presented. Finally, in Sec. IV, future plans are outlined.

\section{SET-UP FOR THE TESPEL INJECTION SYSTEM}

\section{A. W7-X stellarator}

$\mathrm{W} 7-\mathrm{X}$ is an optimized stellarator that was commissioned at the end of $2015^{7}$. Its main mission is to achieve steady-state operation at high normalized pressure with a volume-averaged beta of about $5 \%$, ion temperatures of several $\mathrm{keV}$ and densities $\geq 10^{20} \mathrm{~m}^{-3}$, in order to demonstrate the viability of stellarators as future fusion reactors. Its major radius is $5.5 \mathrm{~m}$, its average minor radius is $0.53 \mathrm{~m}$ and the plasma volume is about 30 $\mathrm{m}^{3}$. Electron cyclotron resonance heating will be used to achieve steady-state heating. This system is formed by 10 gyrotrons, with a total output power of $7.5 \mathrm{MW}$, operating at $140 \mathrm{GHz}$, the 2nd harmonic of the electron cyclotron resonance at $2.5 \mathrm{~T}^{8}$. In addition, two NBI systems, with maximum pulse length of $10 \mathrm{~s}$, will provide $7 \mathrm{MW}$ of total neutral beam power. Recently, inertially cooled island test divertors were installed for the OP1.2a experimental campaign to ensure good pumping and controlled plasma-wall interactions. The island divertor was 
implemented by creating large $5 / 5$ islands at the plasma boundary that intersect the divertor plates.

It is expected to inject the first TESPELs into the W7-X plasma during its OP1.2b operation phase that is planned for mid to late 2018 .

\section{B. W7-X TESPEL Injector}

The design of the TESPEL injector for W7-X (c.f. Fig. 1) is based on the TESPEL system developed for the $\mathrm{LHD}^{4}$. It consists of a motorized storage disk with 60 chambers for TESPELs, a fast electromagnetic gas propulsion valve for pellet acceleration, straight guide tubes with breaks between them to transfer the accelerated TESPEL to the W7-X vacuum vessel, three gas expansion chambers, a dual light-gate system, several vacuum bellows, a Y-adapter (enabling simultaneous injection and observation) and several pneumatically operated vacuum valves. The complete system is mounted on an inclined support structure so that the TESPEL flight path is directed towards the plasma axis. A schematic sketch of the system is shown in Fig. 1. It should be noted that the overall length, width and height of the system are defined by neighboring diagnostic systems (laser blow off and electron cyclotron emission diagnostics $)^{9,10}$, by access restrictions and by torus hall structures. In its final design, the TESPEL storage disk to first light-gate distance is $2.33 \mathrm{~m}$ and the first light-gate to plasma edge (standard magnetic configuration) distance is $2.73 \mathrm{~m}$.

When undertaking experiments, the storage disk can be filled with 700 and/or $900 \mu \mathrm{m}$ diameter TESPELs. Then, since typically $20-30 \mathrm{ml}$ of helium at a pressure of 35 bar is needed to accelerate a pellet to between 300 and $500 \mathrm{~m} / \mathrm{s}$, a stepwise reduction of the resultant increased gas pressure is required in order to prevent gas reaching the $\mathrm{W} 7-\mathrm{X}$ vacuum vessel. This is achieved by having breaks, or jumps between the guide tubes (c.f. Tab. II). These breaks are located within the first two expansion chambers and at gate valve (GV1). The expansion chambers are each fitted with a separate pumping system (c.f. Tab. I). The first expansion chamber (EC 1), i.e., the one nearest the storage disk, is equipped with a helical grooved pump, due to the high gas pressure in this chamber, while the other two chambers are equipped with standard turbo-molecular pumps. In each case, a dry scroll mechanical pump is used as a backing pump. It should be noted that due to space limitations, which has prevented its relocation or reorientation, it has been necessary to provide soft iron shielding about the helical groove pump in order to reduce the local magnetic field to below at least $4.5 \mathrm{mT}$ (Note: manufacturer recommended value is $3.0 \mathrm{mT}$ ) in order to avoid excess heating due to eddy currents in the rotor ${ }^{11}$. Next, a vacuum bellows is positioned between the light gates and the W7$\mathrm{X}$ port (AEE41) for the easy installation of the TESPEL injection system. Finally, a specially designed Y-adapter, that also includes an optical viewport, permits attaching
TABLE I. Specifications of the differential pumping system. * The given volume means the overall volume of the related expansion chamber, including the attached pumping tubes.

\begin{tabular}{|c|c|c|c|}
\hline $\begin{array}{l}\text { expansion } \\
\text { chamber }\end{array}$ & $\begin{array}{l}\text { volume* }^{*} \\
\text { (litres) }\end{array}$ & pump type & $\begin{array}{l}\text { pumping sped } \\
\text { for } \mathrm{He}(\mathrm{l} / \mathrm{s})\end{array}$ \\
\hline EC 1 & 63.5 & $\begin{array}{c}\text { Osaka Vacuum } \\
\text { TS50CW }\end{array}$ & 20 \\
\hline EC 2 & 61.9 & $\begin{array}{c}\text { Pfeiffer Vacuum } \\
\text { HiPace } 300\end{array}$ & 260 \\
\hline EC 3 & 57.5 & $\begin{array}{c}\text { Pfeiffer Vacuum } \\
\text { HiPace } 400\end{array}$ & 470 \\
\hline
\end{tabular}

TABLE II. Barrel and guiding tube dimensions

\begin{tabular}{lcccl}
\hline \hline tube & $\begin{array}{c}\text { length } \\
(\mathrm{mm})\end{array}$ & $\begin{array}{c}\text { outer dia- } \\
\text { meter }(\mathrm{mm})\end{array}$ & $\begin{array}{c}\text { inner dia- } \\
\text { meter }(\mathrm{mm})\end{array}$ & $\begin{array}{l}\text { gap to next } \\
\text { tube }(\mathrm{mm})\end{array}$ \\
\hline barrel & 408.0 & 3.0 & 1.00 & $\approx 5$ \\
GT 1 & 508.0 & 3.18 & 2.18 & $\approx 5$ \\
GT 2 & 721.5 & 6.35 & 4.35 & $\approx 28$ \\
GT 3 & 625.2 & 9.53 & 7.53 & - \\
\hline \hline
\end{tabular}

the TESPEL system to the W7-X. The viewport is oriented so that the line-of-sight of the observation optics intersects the TESPEL flight path at approximately 0.1 $\mathrm{m}$ inside the plasma low field edge. From pellet ablation modelling, using equations within refs. 6,12 with temperature $(\mathrm{Te}(0)=7.4 \mathrm{keV})$ and density $(\mathrm{Ne}(0)=$ $8 \times 10^{19} \mathrm{~m}^{-3}$ ) profiles calculated with the NTSS-code ${ }^{13}$, it is predicted that a TESPEL will be fully ablated after penetrating $0.1 \mathrm{~m}$ inside the plasma.

\section{TESPEL diagnostics}

The W7-X TESPEL injector is equipped with an inline dual light-gate system (separation between the lightgates is $110 \mathrm{~mm}$ ) for obtaining time-of-flight information. As seen in Figure 1, the system is located just after the 3rd guiding tube. Thus, the TESPEL speed - in free flight condition before entering the W7-X vacuum vessel - can be evaluated. The dual light-gate system employs two HeNe lasers ( $5 \mathrm{~mW}$ each), fiber optic cables and light detectors (photo diodes). In addition, dedicated plasma diagnostic systems, collecting light through a viewport mounted on the same vacuum vessel port (Y-adapter) as the TESPEL injector, follow the ablation of the tracerloaded polystyrene pellet as it crosses the plasma and the exposure of the tracer element to the plasma (Note: the viewport to plasma edge distance is $2.35 \mathrm{~m}$ ). Outside the viewport, a beam splitter divides the light. Part of the light passes through the beam splitter to a collimating lens that focuses the light onto an optical fiber for its transfer from the W7-X experimental hall to a laboratory located approximately $40 \mathrm{~m}$ away. The light reflected by the beam splitter will be directed onto the lens of a fiber optic light guide, leading to a fast-frame imaging camera. 


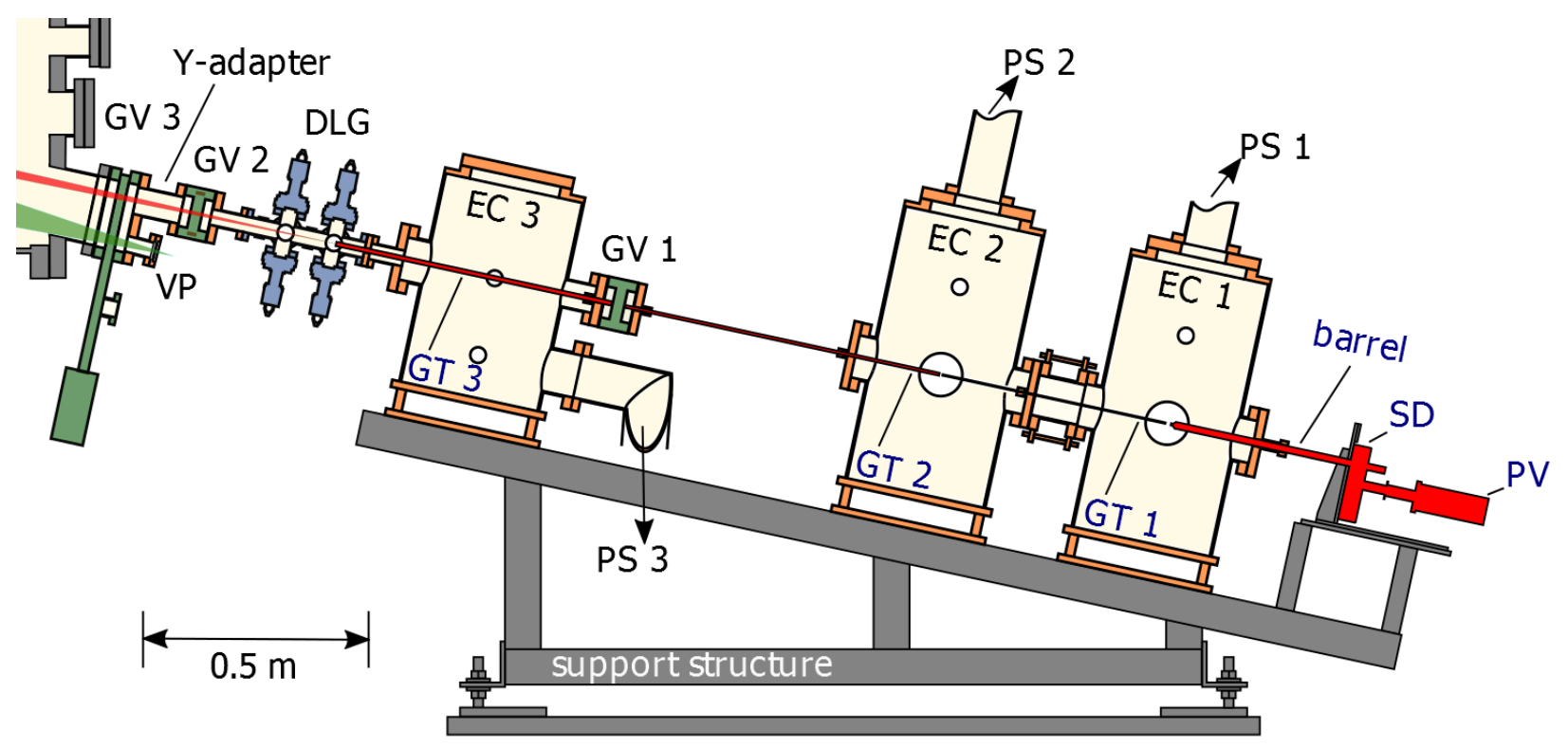

FIG. 1. Schematic drawing of the TESPEL system mounted on its support structure and attached to the W7-X. The principal components are highlighted. EC 1 .. EC 3: expansion chambers; PS 1 .. PS 3: pumping systems (c.f. Tab. I); GV 1 .. GV 3: gate valves; DLG: dual light-gate; VP: viewport; components of the actual injection system PV: propulsion valve; SD: TESPEL storage disk; GT 1 .. GT 3: guiding tubes (c.f. Tab. II)

In the laboratory, the transmitted light is divided again using a fiber optic fan-out. Part of this light is directed to the input slit of a high-resolution spectrometer, equipped with a photomultiplier tube. The remaining light is transferred to a filterscope system, equipped with light filters and photomultiplier tubes. The atomic elements of interest as tracers for the first stage of impurity transport research in $\mathrm{W} 7-\mathrm{X}$ are titanium, vanadium, iron, molybdenum, and tungsten. The inherent elements are hydrogen and carbon from the TESPEL shell. Thus, the filterscope is equipped with seven narrow band-pass filters, centered on intense emission lines from the atomic state of these tracers as well as from neutral hydrogen and carbon (from the polystyrene). The selected spectral emission line wavelengths and transmission details of the selected filters are shown in Table III. Finally, the transmitted light is detected by photomultiplier tubes with spectral response between 185 and $900 \mathrm{~nm}$. Hence, knowing the TESPEL pellet ablation light distribution as a function of time along a discharge (and its speed from the light-gates), the signal traces from this system permit determining the radial positions for shell and tracer depositions. Moreover, it is possible to determine if the tracer deposition is well localized or if the tracer was lost during its flight.

As stated previously, part of the light emitted by the ablating pellet and transmitted through the optical viewport will be directed, using a beam splitter, towards an ultra fast-frame imaging camera (Photron Fastcam SA5, up to $600 \mathrm{kHz}$ ). Moreover, a second fast frame camera (5$10 \mathrm{kHz}$ ) will also view the pellet/plasma interaction from a nearby tangential viewport ${ }^{15}$. In this way it will be
TABLE III. TESPEL shell and selected tracer impurity elements, identified spectral emission lines ${ }^{14}$, plus the central wavelength and the bandpass width of filters used in the filterscope. ${ }^{*}$ There are multiple lines C I around $711 \mathrm{~nm}$.

\begin{tabular}{rllcc}
\hline \hline $\begin{array}{r}\text { Atomic } \\
\text { Element }\end{array}$ & $\begin{array}{l}\text { Emission Line } \\
\text { Wavelength } \\
(\mathrm{nm})\end{array}$ & \multicolumn{3}{c}{$\begin{array}{c}\text { Central Wavelength; } \\
\text { Bandwidth of Filter } \\
(\mathrm{nm})\end{array}$} \\
\hline C I & $711^{*}$ & 710 & $+3 /-0 ;$ & $10+/-2$ \\
H I & 656.3 & 656.3 & $+0.2 /-0 ;$ & $1+/-0.2$ \\
Ti I & 546 & 546.1 & $+1 /-0 ;$ & $5+/-1$ \\
V I & 617 & 620 & $+3 /-0 ;$ & $10+/-2$ \\
Fe I & 628.1 & 630 & $+3 /-0 ;$ & $10+/-2$ \\
Mo I & 550.6 & 550 & $+3 /-0 ;$ & $10+/-2$ \\
W I & 540.5 & 540 & $+3 /-0 ;$ & $10+/-2$ \\
\hline \hline
\end{tabular}

possible to obtain images of the TESPEL ablation cloud and of its detached drifting cloud as it expands along the magnetic field lines. However, it is expected that outward drifting of detached impurity tracer clouds will be significantly reduced compared to hydrogen clouds due to the higher atomic mass of tracer elements, as seen in TJ-II ${ }^{6}$.

\section{TESPEL injector operation}

Preventing unwanted distortions due to transfer of excessive amounts of the propellant gas (helium) into the $\mathrm{W} 7-\mathrm{X}$ vacuum vessel is essential for proper plasma operation. For the most part, this is guaranteed by the dif- 
ferential pumping system of the three expansion chambers. While the main gate valve to $\mathrm{W} 7-\mathrm{X}(\mathrm{GV} 3)$ is controlled by the $\mathrm{CoDaC}$ central operation management and will be kept open during an experimental day, the gate valves GV 1 and GV 2 are directly managed by the injector control system (PLC $+\mathrm{PC})$. It generates individually delayed triggers by using centrally-provided standard triggers ( $\mathrm{T} 0$ and $\mathrm{T} 1$ ). The two gate valves $\mathrm{GV}$ 1 and GV 2 will be opened about ten seconds before each planned TESPEL injection and be closed immediately and sequentially after a TESPEL has passed at each valve position.

As a TESPEL might hit and impair inner-vessel components when it is not completely ablated by plasma, an optically triggered interlock-circuit will be used to prevent such situations. This circuit measures the line-ofsight integrated light and is coupled to the gas propulsion valve (PV) so that it can be activated only when a discharge is safely generated. The activation timing of the valve $\mathrm{PV}$ is also governed by the injector control system.

\section{LABORATORY TESTS}

The TESPEL injection system has been assembled on a laboratory test-bed and tested for correct operation before its transfer to the W7-X experimental hall. For this purpose, the test chamber, containing a target holder (c.f. Fig. 2), was installed instead of the light-gates at the downstream end of the guide tubes. Inside the CF40cross of the test chamber an aluminium tube with a 3 $\mathrm{cm}$ wide cut-out, wrapped with a strip of standard aluminium foil as target (c.f. Fig. 2, right image) was placed. The distance between the third guiding tube and the target foil was $185 \mathrm{~mm}$. This foil was removed from the vacuum system after each test shot to check for pellet arrival. For these tests, solid polystyrene pellets with diameters of 700 and $900 \mu \mathrm{m}$ were used.

In a first series of shots we initially obtained good results for the first 3-4 shots. However, for subsequent shots, it was found that pellet fragments were hitting the target, see for example, shot \#6 in Fig. 3. This result pointed to a possible misalignment of the guiding tubes. By illuminating the whole line-of-flight along the barrel and the tubes by means of a bright halogen lamp and taking pictures with a camera attached as close as possible to the end of the last guiding tube, the tube alignment could be imaged. Hence, the problem had been localized and solved by readjusting one of the support flanges of the guiding tubes. This led to a significantly better concentric alignment of the guiding tubes (c.f. Fig. 4). The following ten test injections were all successful, resulting in impact images as shown for sample \#20 in Fig. 3. It should be noted that beside the realignment of the guiding tubes for the second injection series, an additional blank shot was made after each pellet injection to blow out dust particles which might have remained from collisions of the pellet with the tube walls.

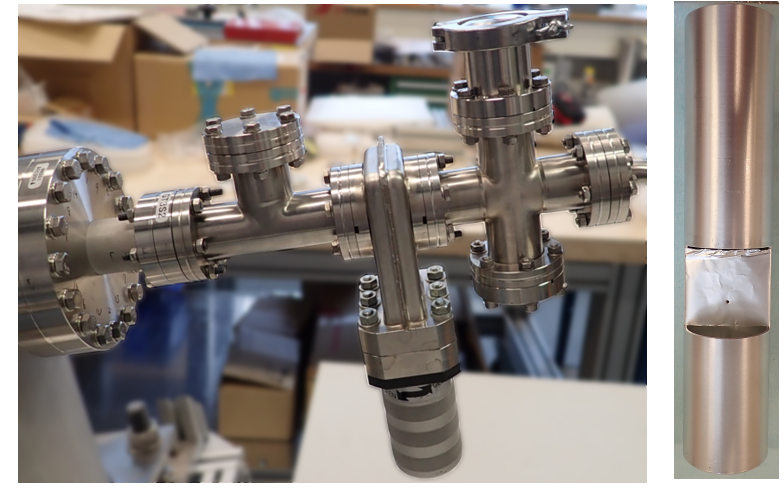

FIG. 2. Photo of the test chamber (left) for the target holder (to the right of the vacuum valve) and of the target holder (right) with attached aluminium test stripe after a successful pellet transfer.
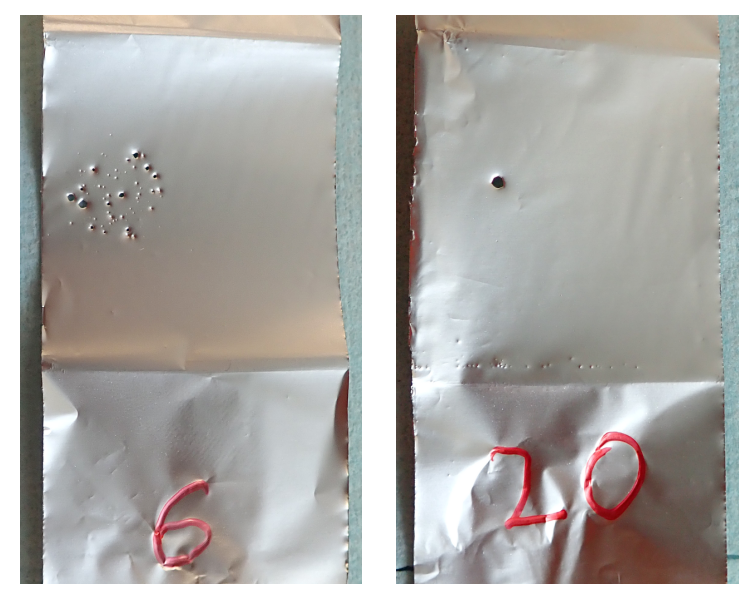

FIG. 3. Representative examples of impact images during the first test series (\#6, left) where only pellet fragments had hit the target and of the second test series (\#20, right) where all pellets had been transfered intact.

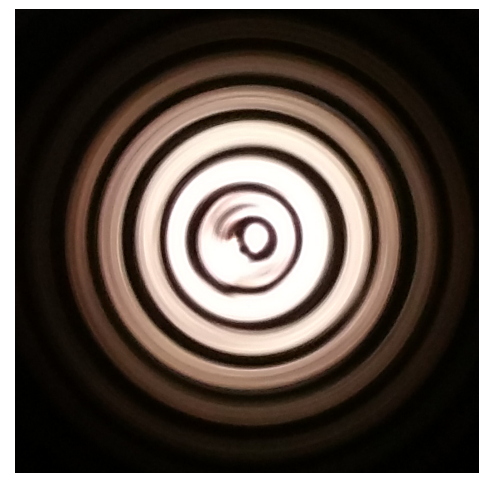

FIG. 4. Image taken from the end of the third guiding tube after successful readjustment of the tubes. In the picture, a bright spot, from the lamp located outside the barrel, is surrounded by concentric rings. The first broad, slightly irregular ring structure around the central spot is associated with reflections within the second guiding tube whereas the multitude of following rings belongs to the third tube. 


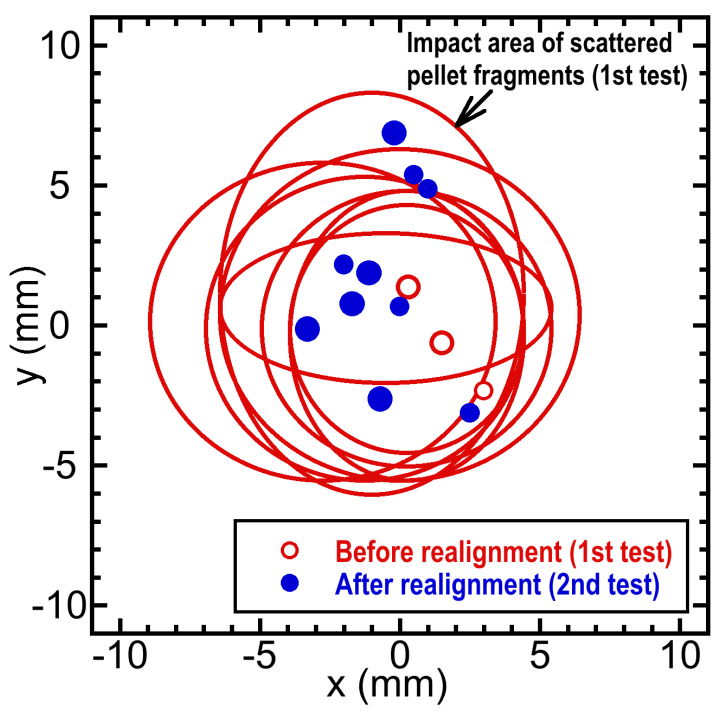

FIG. 5. Pellet or fragment distribution (relative to their center of gravity) in the two test series. The coordinates refer to the horizontal $(\mathrm{x})$ and vertical direction $(\mathrm{y})$ on the target foil in Fig. 3. The radii of the ellipses and the markers indicate the spatial distribution of the pellet fragments and the impact holes for intact pellets, respectively.

Figure 5 summarizes the results of the two test series. As TESPELs experience a number of bounces off the inner tube walls during their flight, each successfully transferred pellet leaves the final tube at a different angle (within the stray angle cone) and hits a different region on the target foil in which it creates a single hole (solid markers). In case of a seriously misaligned guiding tube, the number of reflections increases strongly or the pellet might even hit the edge of the subsequent tube which leads to fragmentation. The single fragments collide with the tube walls, each under a different angle, and hit the target foil over a broad distribution. The ellipse radii indicate the impact area of scattered pellet fragments. In the first test series, three intact pellets arrived at the target foil (small red circles). Taking the diameter of the ellipses and the distance between the exit of the last guiding tube and the sample foil to calculate the maximum stray angle (full angle) results in $\alpha_{x}=3.9^{\circ}$ and $\alpha_{y}$ $=4.5^{\circ}$.

For the second test series, the diameter of the markers indicates the initial pellet diameter which almost corresponds to the diameter of the impact hole on the target foil. Using the maximum deviation from the center of gravity of the impact hole distribution to calculate the pellet stray angle gives $\alpha_{x}=1.8^{\circ}$ and $\alpha_{y}=3.1^{\circ}$.

\section{CONCLUSIONS AND OUTLOOK}

A new TESPEL pellet injection system has been designed and built for the W7-X. During laboratory on a test-bed, successful transfer of pellets was achieved between the storage disk and a target foil located on the downstream end, once good guide tube alignment was attained. For this, 700 and $900 \mu \mathrm{m}$ pellets were used and ten out of ten pellets were transferred completely intact.

The TESPEL injection system will be installed on the W7-X before mid 2018. Commissioning will begin with a set of blank shots into vacuum (without plasma) to determine the amount of residual propellant gas entering the W7-X vacuum vessel and minimize it by adjusting the timing to close the gate valves GV1 and GV2. Subsequently, blank shots and TESPEL injections will be performed to assess the impact on the plasma, to adjust the signal levels in the filterscope, the threshold of the optical interlock circuit (used to prevent injections without plasma) and to optimize the amount of tracer particles, loaded into the TESPELs.

The OP1.2b operational phase of W7-X will commence in the summer of 2018 and continue until mid fall - with first scientific results from TESPEL injections expected towards the end of OP1.2b.

\section{ACKNOWLEDGMENTS}

This work has been carried out within the framework of the EUROfusion Consortium and has received funding from the Euratom research and training programme 2014-2018 under grant agreement No 633053. The views and opinions expressed herein do not necessarily reflect those of the European Commission. This work is supported by the Program for Enhancing Research Collaboration (UFEX105), the Young Researchers Supporting Program (UFEX106), the NIFS/NINS under Strategic International Research Exchange Promotion Program (UFEX402), and the NINS program of Promoting Research by Networking among Institutions (Grant No. 01411702). KJM receives support for this project from the Spanish MINECO (FIS2017-89326-R).

\footnotetext{
${ }^{1}$ S. Sudo, J. Plasma Fusion Res. 69, 1349 (1993).

${ }^{2}$ S. Sudo and N. Tamura, Rev. Sci. Instrum. 83, 023503 (2012).

${ }^{3}$ S. Okamura et al., Nucl. Fusion 39, 1337 (1999).

${ }^{4}$ S. Sudo et al., Plasma Phys. Control. Fusion 55, 095014 (2013).

${ }^{5}$ N. Tamura et al., Rev. Sci. Instrum. 87, 11D619 (2016).

${ }^{6}$ K. J. McCarthy et al., Europhys. Lett. 120, 25001 (2017).

${ }^{7}$ R. Wolf et al., Nucl. Fusion 57, 102020 (2017).

${ }^{8}$ V. Erckmann et al., Fusion Sci. Technol. 52, 291 (2007).

${ }^{9}$ E. Pasch et al., Rev. Sci. Instrum. 87, 11E729 (2016).

${ }^{10} \mathrm{H}$. Thomsen et al., J. Instrum. 10, P10015 (2015).

${ }^{11}$ A. Nishidé et al., J. Vac. Sci. Technol. 20, 1105 (1982).

${ }^{12}$ K. V. Khlopenkov and S. Sudo, Rev. Sci. Instrum. 69, 3194 (1998).

${ }^{13}$ Y. Turkin et al., Phys. Plasmas 18, 022505 (2011).

${ }^{14}$ R. Payling and P. Larkins, Optical Optical Emission Lines of the Elements (John Wiley \& Sons, Ltd., Chichester,, 2000).

${ }^{15}$ G. Kocsis et al., Fusion Eng. Design 96-97, 808 (2015).
} 